\title{
Diagnosis and treatment of uncommon ileal endometriosis: a case report and literature review
}

\author{
M. Mabrouk ${ }^{1,2}$, D. Raimondo ${ }^{1}$, M. Cofano ${ }^{1}$, L. Cocchi ${ }^{1}$, R. Paradisi ${ }^{1}$, R. Seracchioli ${ }^{1}$ \\ ${ }^{1}$ Division of Gynaecology and Human Reproduction Physiopathology, Department of Medical and Surgical Sciences \\ (DIMEC), IRCCS Azienda Ospedaliero, Univeristaria di Bologna. S. Orsola Hospital, University of Bologna, Via Mas- \\ sarenti 13, Bologna, 40138 Italy; ${ }^{2}$ Cambridge Endometriosis \& Endoscopic Surgery Unit (CEESU) and Cambridge \\ University Hospitals NHS Foundation Trust, Addenbrooke's Hospital, Cambridge, United Kingdom.
}

Correspondence at: Laura Cocchi, MD. Gynaecology and Human Reproduction Physiopathology, DIMEC, S. Orsola Hospital, University of Bologna, Massarenti, 13 - 40138 Bologna, Italy. Tel: + 39051 2144389; Fax: +39 0516364392 ; E-mail: laura.cocchi4@gmail.com

\begin{abstract}
Endometriosis is defined as the presence of endometrial tissue outside the uterine cavity. It is a common finding in premenopausal women and commonly affects the gastrointestinal tract, especially the rectosigmoid tract. Small bowel involvement is rare and usually asymptomatic making diagnosis difficult. Here we report an uncommon case of exophytic ileal endometriosis surgically treated. Detailed pre-operative counselling on the risk of ileal surgery should always be considered in all cases with endometriosis requiring surgery. We also present a review of the literature regarding the clinical presentation, diagnosis, and treatment of this challenging condition.
\end{abstract}

Key words: ileal endometriosis, endometriosis, small bowel endometriosis, laparoscopic surgery, laparoscopy.

\section{Introduction}

Endometriosis is a chronic, inflammatory, oestrogen-dependent disease defined as the presence of endometrial glands and stroma outside the uterine cavity and it affects up to $15 \%$ of women during reproductive age (Olive and Pritts, 2001). The most frequent implants are the ovaries, uterosacral ligaments, cul-de-sac, vagina, urinary tract, and bowel. (Chapron et al., 2006; Chapron et al., 2003) In women with endometriosis, bowel involvement is relatively common (3-12\%) and the most frequent localisations occur in the rectum and rectosigmoid junction (50-90\%), the small bowel (2-16\%), appendix (3-18\%) and caecum (2-5\%). The ileum is affected in $4 \%$ of women with bowel endometriosis, (Chapron et al., 2006) frequently associated with rectosigmoid lesions.

Women with isolated ileal endometriosis are usually asymptomatic or have non-specific symptoms: abdominal pain, bloating and cramps, altered bowel habits (constipation and/or diarrhea), dyspareunia and haematochezia (Rodriguez-Lopez et al., 2018). There is no high-precision imaging test for ileal endometriosis (Tong et al., 2013). Diagnosis of ileal endometriosis is usually made incidentally during surgery for other endometriosis sites or following direct complications of ileal involvement: bowel obstruction, ileocecal intussusception or perforation (Rodriguez-Lopez et al., 2018). At laparoscopy, typical ileal lesions are nodular in shape with transmural and endophytic growth and are often located on the ileocecal junction (Ruffo et al., 2011).

Here we present, for the first time, an asymptomatic exophytic nodule of the distal ileum as an uncommon case of ileal endometriosis.

\section{Case history}

A 41-year-old multiparous woman presented to our centre with a history of severe dysmenorrhea, dyspareunia and catamenial dysuria within the last six months resistant to progestin therapy. She also reported intermittent perimenstrual intestinal bloating and constipation. She had 
recently undergone a diagnostic laparoscopy in another hospital and was diagnosed with bladder endometriosis. Gynaecological examination revealed moderate pain on anterior and posterior vaginal fornix palpation. Transvaginal sonography (TVS) and magnetic resonance imaging (MRI) showed the presence of adenomyosis and a deep endometriotic nodule of the left uterosacral ligament (USL) (17 $\mathrm{mm}$ mean diameter) and bladder dome (25 mm mean diameter).

Surgery was planned for the excision of the endometriotic nodule by partial cystectomy and removal of the affected left USL. Because of her intestinal symptoms, the woman was informed and gave informed consent about the risk of bowel surgery, including small bowel and appendix procedures.

At diagnostic laparoscopy we systematically perform, before inspecting the pelvis, an assessment of the abdomen, including appendix, ileocaecal junction, and distal ileum. During the inspection, a $4 \mathrm{~cm}$ round, exophytic mass with a translucent-blue and smooth surface covered with small nodules on the distal ileum was found (Figure 1). Ileal surgery was performed by an experienced multidisciplinary team with general surgeon and urologist. After removal of the pelvic lesions, mobilisation of the caecum and distal ileum a transverse suprapubic minilaparotomy were performed to externalise the ileum.
The affected bowel tract was isolated and resected, and side-to-side anastomosis was performed with the GIA75 device. The postoperative course was regular. Pathological examination confirmed a 4-cm exophytic ileal endometriotic implant without atypical features and endometriosis of the appendix. At 6-month follow-up, pain and bowel symptoms markedly improved. The women provided informed consent to report data and images of the case.

\section{Review of literature and Discussion}

We performed a narrative review on endometriosis diagnosis and management. To determine eligible articles, the following electronic databases were screened from inception to January 2021: PubMed, Scopus, and Embase. To retrieve articles related with the theme of interest, the following terms were used to search the electronic databases: "endometriosis" AND "ile*" OR "small bowel". Reports included in this review consisted of case reports and clinical studies describing management of ileal endometriosis. Two study investigators (M.C. and L.C.) independently conducted the primary literature research using the main search terms.

A total of 12 studies ( 7 case report, 5 clinical studies) were selected and included in this systematic review (Table I).

We found a total of 63 cases of ileal endometriosis. Age ranged from 18 to 61 years.
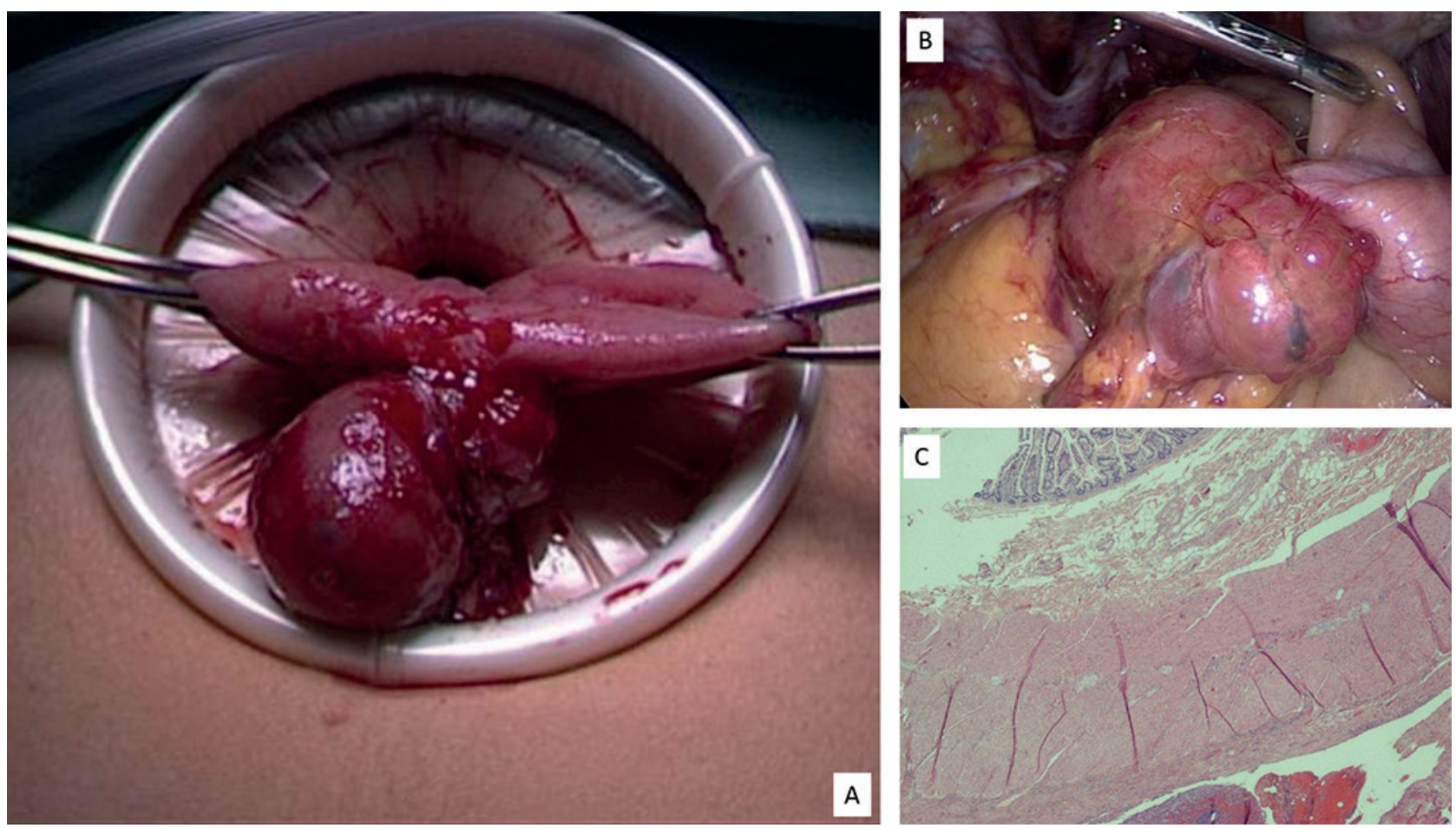

Figure 1: A: Mini-laparotomy with extrusion of the distal ileum affected by an endometriotic nodule. B: Diagnostic laparoscopy showing a $4 \mathrm{~cm}$ round exophytic mass of $4 \mathrm{~cm}$ with a smooth, translucent-blue surface coated by small nodules on the distal ileum. C: Ileal endometriosis, at histological examination, 10x magnification. Note an area of florid endometriosis in the ileal tract serosa. The mucosa with villi is opposite and is not involved by endometriosis and phlogosis. On the serosa there is an intense and active phlogosis with hemorrhagic spread. 
Out of 56 women with available data about surgical history for endometriosis, 37 (66\%) had a previous diagnosis.

Symptoms were variously reported among studies. Particularly, abdominal pain and chronic pelvic pain were the most described ones, occurring in 46/53 $(87 \%)$ women. Bowel symptoms were present in 23/46 (50\%) women. Seven women suffered of small bowel obstruction. Clinical presentation was detailed only in five cases: 3 presented longstanding story of diarrhoea and abdominal pain, while 2 acute onset with abdominal pain and vomiting.

Primary indications for surgery, when reported, were pelvic pain in $38(73 \%)$ women, acute small bowel obstruction in $7(13 \%)$, diagnosis of pelvic deep infiltrating endometriosis (DIE) in $6(12 \%)$, spontaneous enterocutaneous fistula in one $(2 \%)$.

Concerning the preoperative imaging, several imaging modalities were used. Specifically, of the 37 women who underwent computerised tomography (CT), an ileal lesion was suspected in only 3 (8\%) of them. Rousset et al. (2014) diagnosed all 6 cases of ileocaecal endometriotic lesions using 3.0-Tesla (T) MRI enterography; conversely, in Gimonet et al. (2016) no ileal lesion were found using MRI in 6 patients. A preoperative diagnosis of ileal endometriosis was suspected in one $(3 \%)$ of 33 women who had double contrast barium enema (BDCE) and in one of the 9 women (11\%) who underwent colonoscopy.

Diagnosis of ileal endometriosis was made during surgery in 43 women $(43 / 63-68,3 \%)$, using $3.0-\mathrm{T}$ MRI enterography in 6 cases $(6 / 63-9,5 \%)$, using MRI in 4 cases $(4 / 63-6,3 \%)$, using CT in 3 cases $(3 / 63-4,7 \%)$, in 1 case with BDCE $(1,6 \%)$, in 1 case with colonoscopy, and in 1 case only at histological examination.

The presence of other lesions of DIE during surgery was found in 50 of 58 women (86\%).

The surgical approach varies between the studies and were not always specified. Laparoscopic approach was performed in 37 women of $52(71 \%)$, while 15 women underwent laparotomy (29\%).

Only six studies investigated surgical complications. Out of 49 women with available data, 5 cases $(10 \%)$ required blood cell transfusion, one (2\%) reoperation for bleeding, one ureteral fistula, one permanent right femoral nerve lesion and one rectal fistula.

Among women with available follow-up, 95\% (38/40) reported improvement of pain symptoms. In Ruffo et al. (2011) 2 women $(2 / 18,11 \%)$ reported altered bowel habits; in Fedele et al. (2014) two patients $(2 / 8,25 \%)$ complained constipation, two (2/8 - 25\%) alternating constipation and loose stool, and one bloating $(1 / 8-13 \%)$.
Due to the rarity of cases, the literature regarding small bowel endometriosis is limited to a few case reports. The first case of ileal endometriosis was published in 1956 by G. Melody, (Melody, 1956) after which several isolated cases were reported (Table 1).

Bowel endometriosis lesions can result from the implantation of ectopic endometrial cells on the intestinal serosa that progressively invades the bowel wall. Endometriosis infiltrating the muscularis propria of the bowel can lead to localised fibrosis in the bowel wall, stenosis and bowel obstruction (Anaf et al., 2004). Small bowel endometriosis may be suspected in nulliparous fertile women with signs of bowel obstruction, especially during menstruation (Orbuch et al., 2007). However, women with isolated ileal endometriosis may be asymptomatic or present with non-specific/overlapped symptoms.

While the diagnosis of rectosigmoid endometriosis can often be obtained through rectovaginal examination, TVS, transrectal sonography and MRI, the diagnosis of ileal endometriosis was more challenging (Fedele et al., 2014). MRI had the highest sensitivity (77\%-93\%) for the diagnosis of bowel endometriosis, especially for lesions with endophytic growth, however, this sensitivity declined considering only the small bowel (Bazot et al., 2004).

In all cases, particularly in those with nonspecific/overlapped symptoms, careful diagnostic laparoscopy mapping all potential locations of endometriosis in the abdomino-pelvic cavity is essential to prevent repetitive surgeries and relative complications. On abdominal inspection, usually ileal lesions are nodular in shape with transmural and endophytic growth. If small bowel endometriosis is present, laparoscopic ileal/ileocecal resection should be considered part of complete eradication of macroscopic disease, also because this endometriosis is mainly constituted of fibrosis and sclerosis, which do not respond to hormonal treatment (Ruffo et al., 2011).

This is the first reported case of exophytic endometriotic ileal nodule.

\section{Conclusions}

Since most cases of ileal endometriosis are overlooked during preoperative work-up, detailed pre-operative counselling on the risk of ileal/ ileocecal resection is mandatory, in symptomatic cases with endometriosis requiring surgery. Furthermore, having a high level of clinical suspicion for ileal endometriosis is also important to optimise the diagnostic accuracy of imaging technique and diagnostic laparoscopy. 


\begin{tabular}{|c|c|c|c|c|c|}
\hline 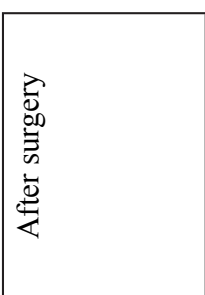 & $\bar{n}$ & 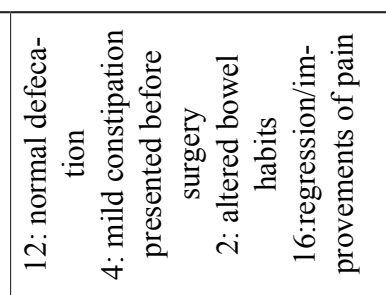 & $\tilde{n}$ & 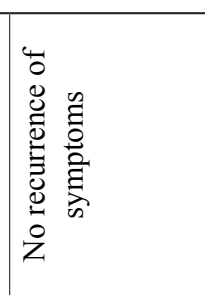 & 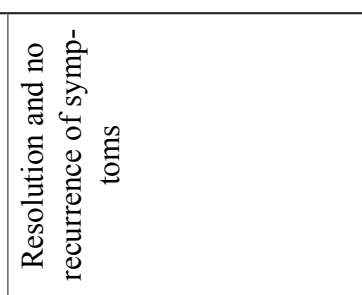 \\
\hline 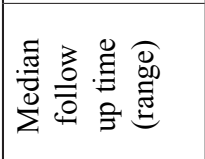 & $\tilde{z}$ & 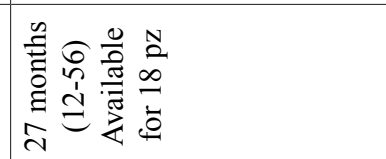 & $\tilde{z}$ & 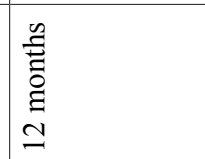 & 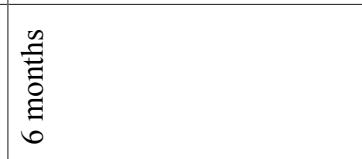 \\
\hline 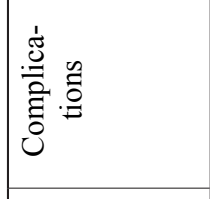 & $\tilde{z}$ & 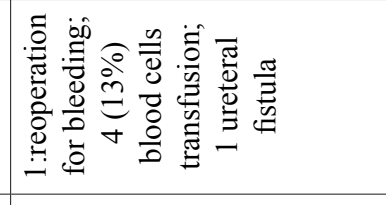 & $\AA$ & $\AA$ & $\tilde{z}$ \\
\hline 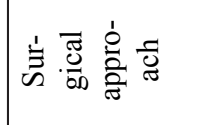 & $\Xi$ & 象高 & $\Xi$ & 矛 & $\frac{n}{z}$ \\
\hline 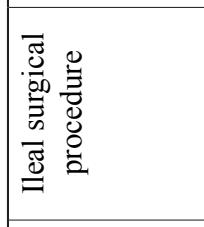 & 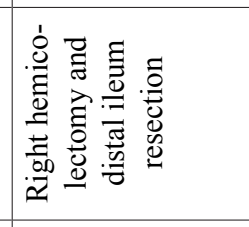 & 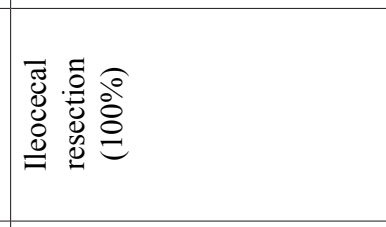 & 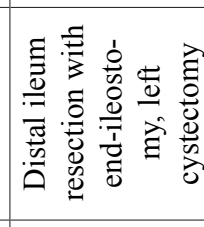 & 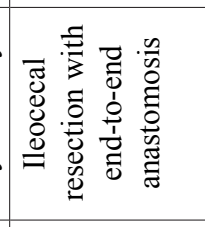 & 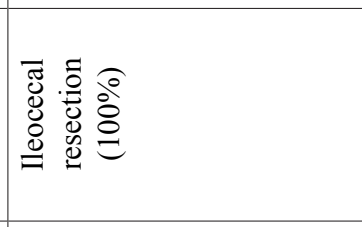 \\
\hline 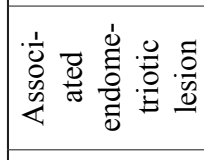 & z & 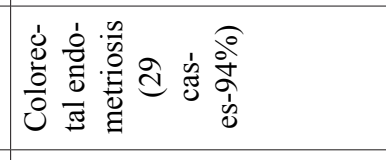 & 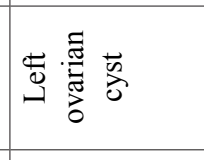 & 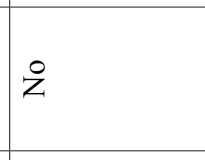 & 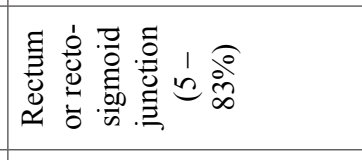 \\
\hline 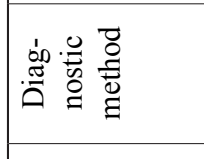 & 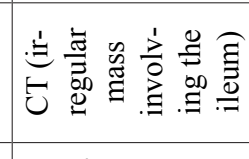 & 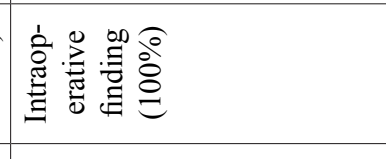 & 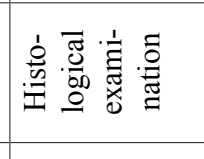 & E & 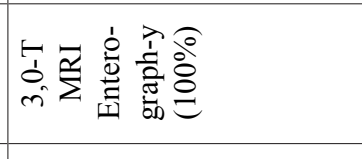 \\
\hline 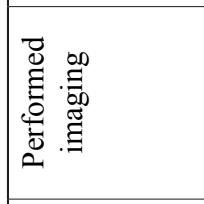 & 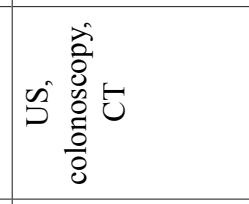 & 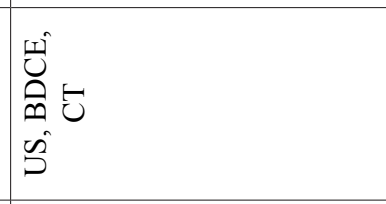 & 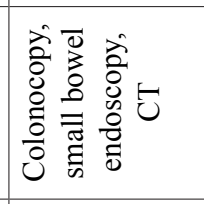 & 它 & 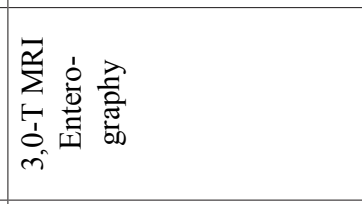 \\
\hline 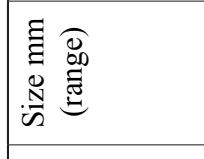 & 言 & $\tilde{z}$ & $\tilde{z}$ & 总高 & $\tilde{z}$ \\
\hline 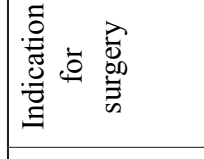 & 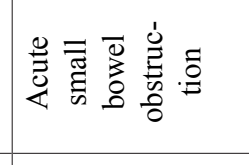 & 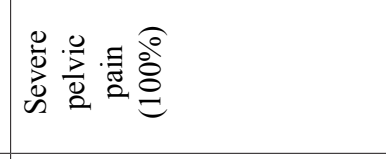 & 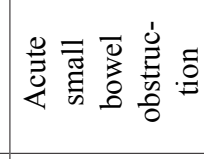 & 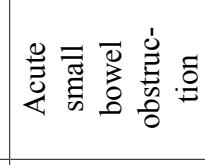 & 产㟋 \\
\hline 高高 & 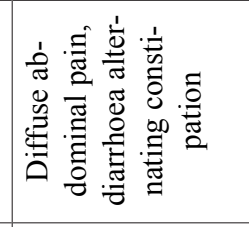 & 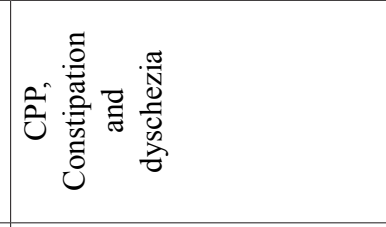 & 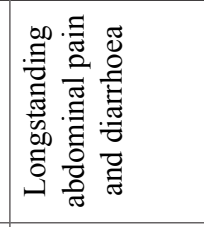 & 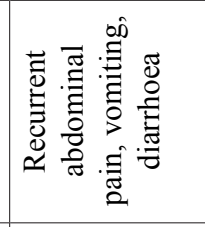 & 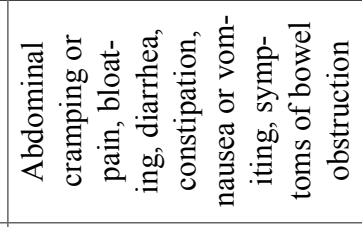 \\
\hline 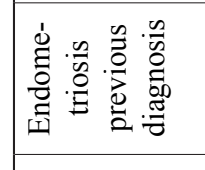 & $\ddot{z}$ & 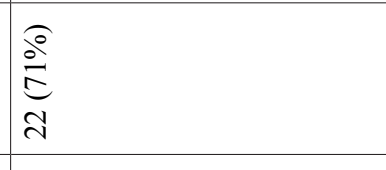 & 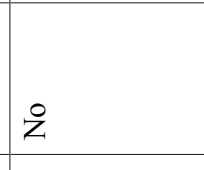 & $\AA$ & $\tilde{z}$ \\
\hline 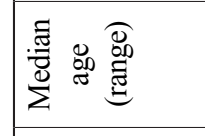 & J & ‡高宗 & $\bar{\lambda}$ & F & $\tilde{z}$ \\
\hline 飔巨 & - & $\bar{m}$ & - & - & 0 \\
\hline 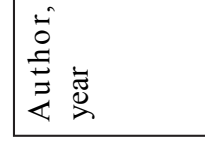 & 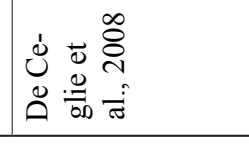 & 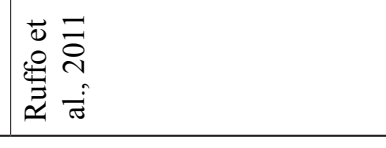 & 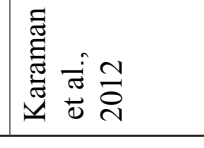 & 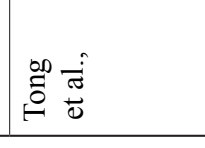 & 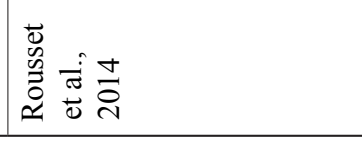 \\
\hline
\end{tabular}




\begin{tabular}{|c|c|c|c|c|}
\hline 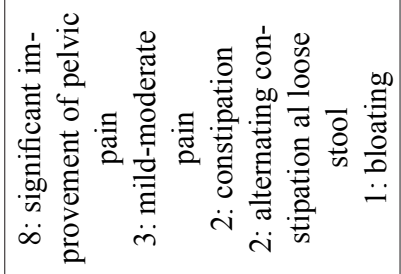 & z & $\tilde{z}$ & $\bar{z}$ & 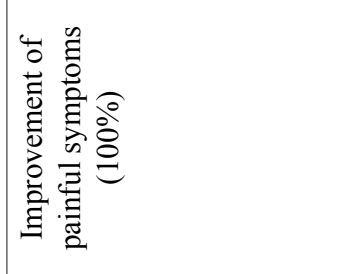 \\
\hline 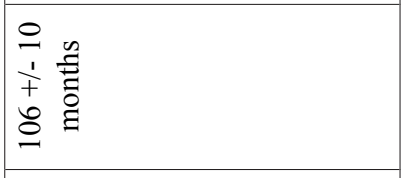 & $\bar{z}$ & $\tilde{z}$ & $\tilde{z}$ & $\tilde{z}$ \\
\hline 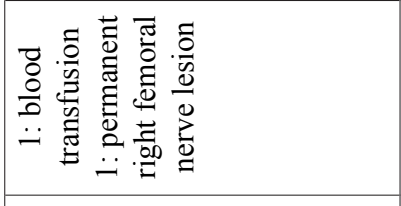 & $\sum_{z}^{2}$ & 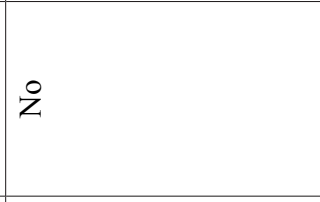 & $\bar{z}$ & 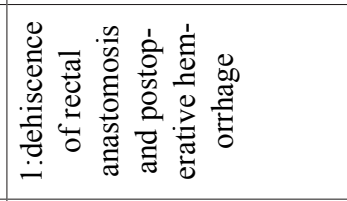 \\
\hline ث용 & $\tilde{z}$ & $\tilde{z}$ & ڤ్ & 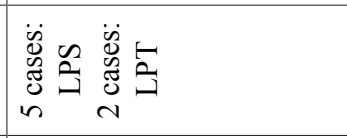 \\
\hline 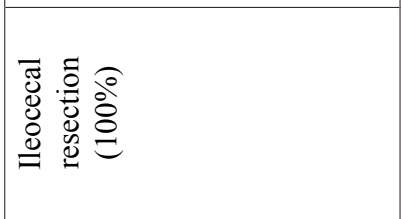 & 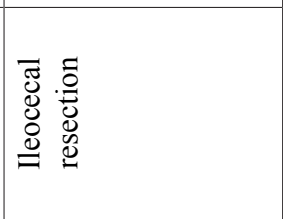 & 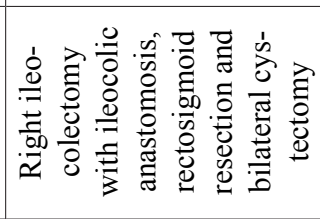 & 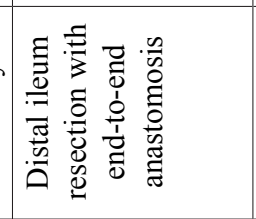 & 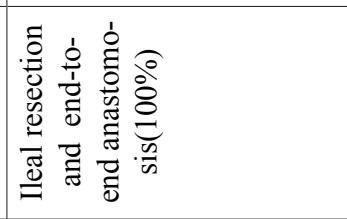 \\
\hline 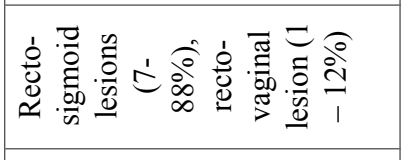 & $\tilde{z}$ & 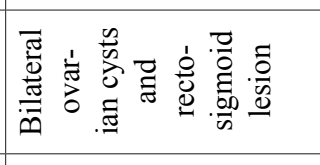 & $\tilde{z}$ & 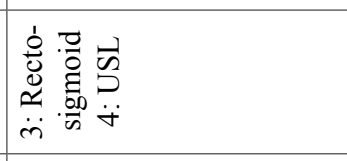 \\
\hline 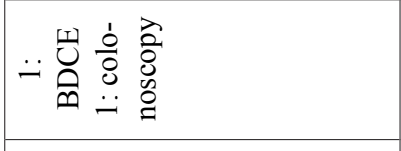 & $\tilde{z}$ & 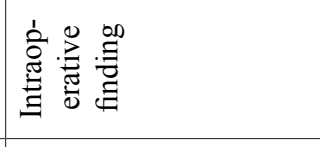 & 主: 总 & 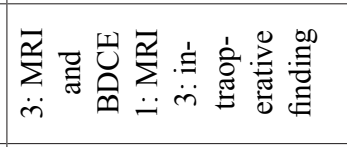 \\
\hline 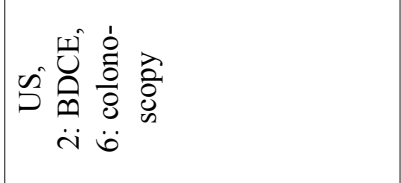 & $\overrightarrow{\tilde{\Sigma}}$ & $\begin{array}{l}\hat{U} \\
\text { vis }\end{array}$ & 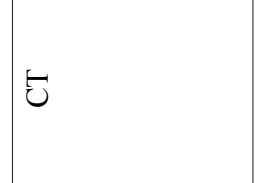 & $\bar{z}$ \\
\hline$\tilde{z}$ & $\bar{z}$ & $\tilde{z}$ & $\tilde{z}^{n}$ & $\tilde{z}$ \\
\hline 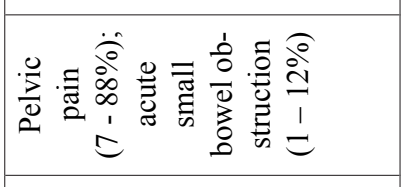 & $\tilde{z}$ & 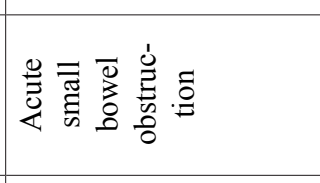 & 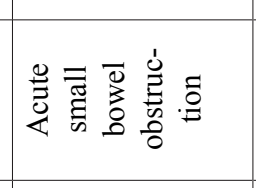 & $\tilde{z}$ \\
\hline 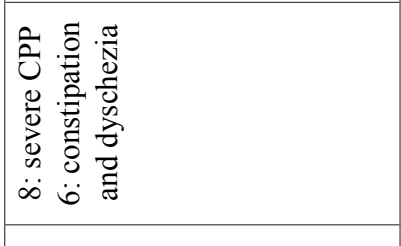 & 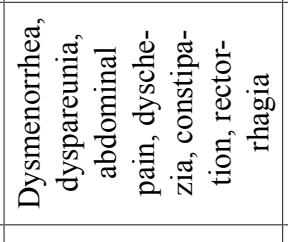 & 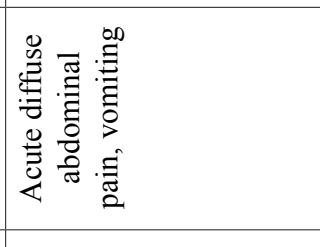 & 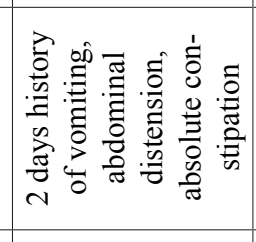 & 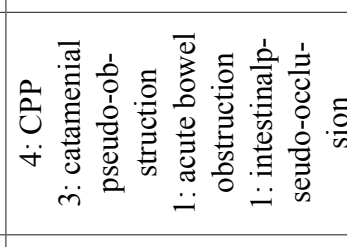 \\
\hline 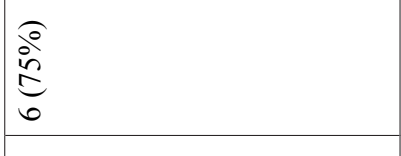 & 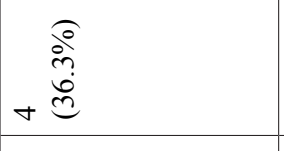 & $\mathscr{\infty}$ & そ & 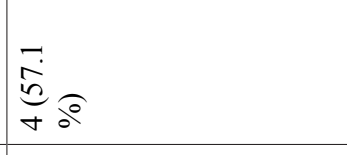 \\
\hline $\begin{array}{l}\text { dy } \\
\text { gे }\end{array}$ & 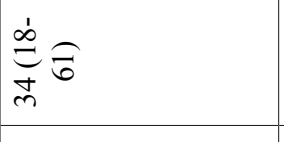 & n & f & 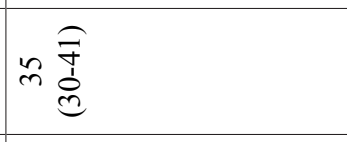 \\
\hline$\infty$ & + & - & - & r \\
\hline 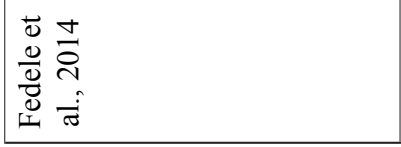 & 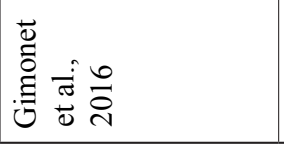 & 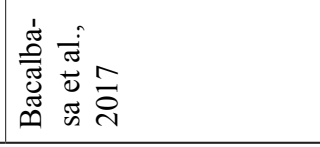 & 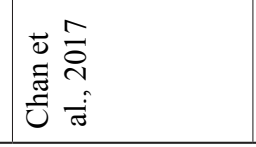 & 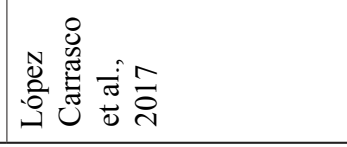 \\
\hline
\end{tabular}




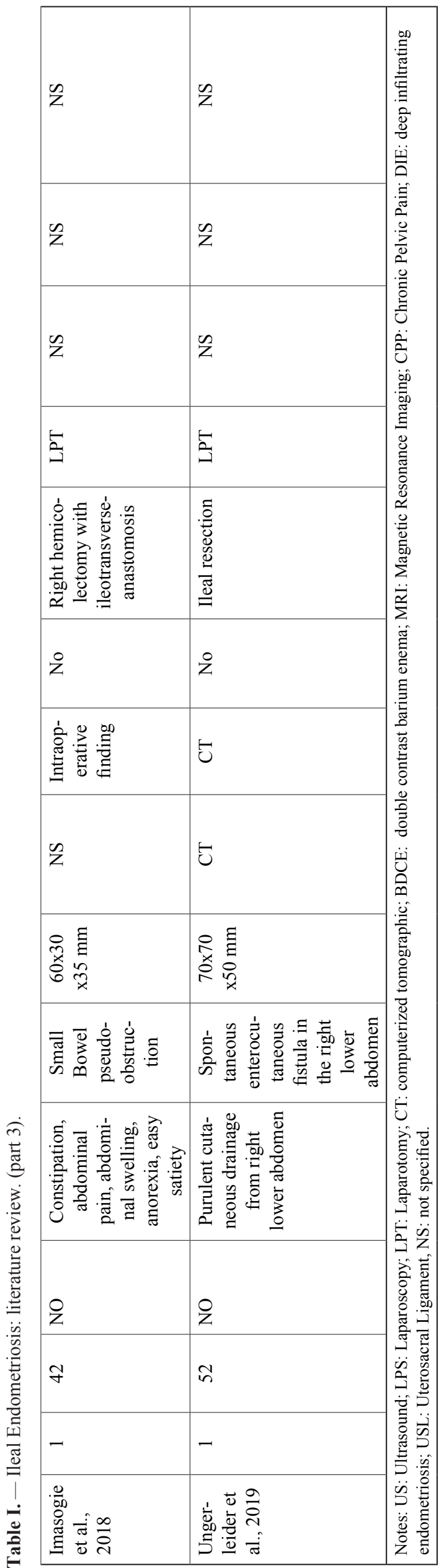

\section{References}

Anaf V, Nakadi I El, Simon P et al. Preferential infiltration of large bowel endometriosis along the nerves of the colon. Hum Reprod. 2004;19:996-1002.

Bacalbasa N, Balescu I, Filipescu A. Ileocecal obstruction due to endometriosis - A case report and literature review. In Vivo (Brooklyn). 2017;31:999-1002.

Bazot M, Darai E, Hourani R et al. Deep pelvic endometriosis: MR imaging for diagnosis and prediction of extension of disease. Radiology. 2004;232:379-89.

De Ceglie A, Bilardi C, Blanchi S et al. Acute small bowel obstruction caused by endometriosis: A case report and review of the literature. World J Gastroenterol. 2008;14:3430-4.

Chan DL, Chua D, Ravindran P et al. A case report of endometriosis presenting as an acute small bowel obstruction. Int J Surg Case Rep. 2017;41:17-9.

Chapron C, Chopin N, Borghese B et al. Deeply infiltrating endometriosis: pathogenetic implications of the anatomical distribution. Hum Reprod. 2006;21:1839-45.

Chapron C, Fauconnier A, Vieira M et al. Anatomical distribution of deeply infiltrating endometriosis: Surgical implications and proposition for a classification. Hum Reprod. 2003;18:157-61.

Fedele L, Berlanda N, Corsi C et al. Ileocecal endometriosis: clinical and pathogenetic implications of an underdiagnosed condition. Fertil Steril. 2014;101:750-3.

Gimonet $\mathrm{H}$, Laigle-Quérat V, Ploteau S et al. Is pelvic MRI in women presenting with pelvic endometriosis suggestive of associated ileal, appendicular, or cecal involvement? Abdom Radiol. 2016;41:2404-10.

Imasogie DE, Agbonrofo PI, Momoh MI et al. Intestinal obstruction secondary to cecal endometriosis. Niger. J Clin Pract. 2018;21:1081-5.

Karaman K, Pala EE, Bayol U et al. Endometriosis of the Terminal Ileum: A Diagnostic Dilemma. Case Rep Pathol. 2012;2012:1-4.

López Carrasco A, Hernández Gutiérrez A, Hidalgo Gutiérrez PA et al. Ileocecal endometriosis: diagnosis and management. Taiwan. J Obstet Gynecol. 2017;56:243-6.

Melody GF. Endometriosis causing obstruction of the ileum. Obstet. Gynecol. 1956;8:468-72.

Olive DL, Pritts EA. Treatment of Endometriosis. N Engl J Med. 2001;345-:266-75.

Orbuch IK, Reich H, Orbuch M et al. Laparoscopic treatment of recurrent small bowel obstruction secondary to ileal endometriosis. J Minim Invasive Gynecol. 2007;14:113-5.

Rodriguez-Lopez M, Bailon-Cuadrado M, Tejero-Pintor FJ et al. Ileocecal intussusception extending to left colon due to endometriosis. Ann R Coll Surg Engl. 2018;100:e62-3.

Rousset P, Peyron N, Charlot M et al. Bowel endometriosis: Preoperative diagnostic accuracy of 3.0-T MR enterography-initial results. Radiology. 2014;273:117-24.

Ruffo G, Stepniewska A, Crippa S et al. Laparoscopic ileocecal resection for bowel endometriosis. Surg Endosc. 2011;25:1257-62.

Tong YL, Chen Y, Zhu SY. Ileocecal endometriosis and a diagnosis dilemma: A case report and literature review. World J Gastroenterol. 2013;19:3707-10.

Ungerleider G, Cheng M, Park C et al. Ileal endometriosis: a rare cause of enterocutaneous fistula. J Gastrointest Surg. 2019;23:1524-6. 